\title{
Positional Isomerism Controlled Electronic and Photochromic Properties of Naphthalene Diimide-Based Chlorozincate Hybrids
}

Pengfei Hao, * Huihui Zhu, Yue Pang, Junju Shen, and Yunlong Fu*

Key Laboratory of Magnetic Molecules \& Magnetic Information Materials Ministry of Education, The School of Chemical and Material Science, Shanxi Normal University, Linfen 041004, China.

\section{Supporting Information (SI)}

\section{Content}

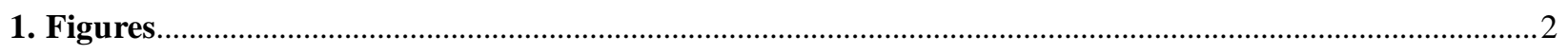

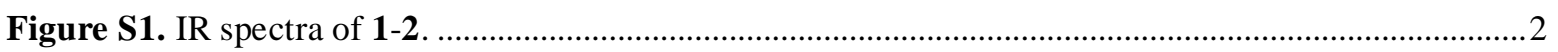

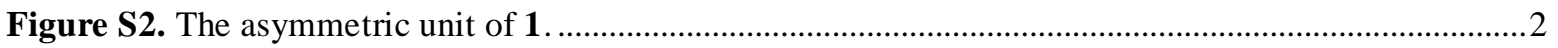

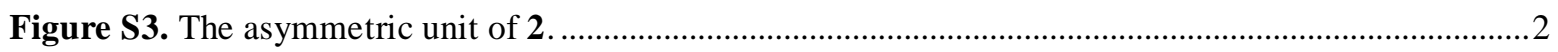

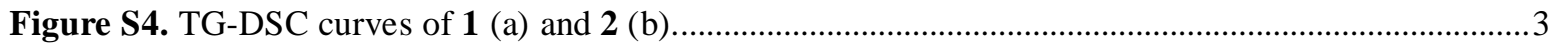

Figure S5. The comparison of energy gaps and UV-vis absorption spectra of $\mathbf{1}$ and $\mathbf{2}$ before irradiation. .3

Figure S6. PXRD patterns for $\mathbf{1}$ and $\mathbf{1 P}$ at room temperature. …….......................................................

Figure S7. UV-vis absorption spectra of 1, 1P-dark and 1P-heating. .......................................................

Figure S8. The switching cycles of coloration-decoloration processes of 1 .................................................

Figure S9. Zn 2p (a), C 1s (b) and O 1s (c) XPS core-level spectra of $\mathbf{1}$ and $\mathbf{1 P}$ at room temperature. ......5

Figure S10. Powder X-ray diffraction (PXRD) patterns for $\mathbf{2}$ and $\mathbf{2 P}$ at room temperature. ......................5

Figure S11. Zn 2p (a), C 1s (b) and O 1s (c) XPS core-level spectra of $\mathbf{2}$ and $\mathbf{2 P}$ at room temperature.....6

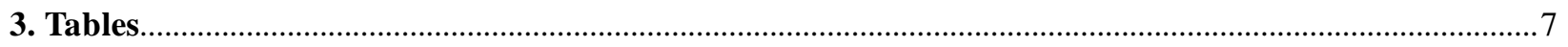

Table S1. Crystallographic data and refinement of compounds 1, 1P and 2 ...........................................

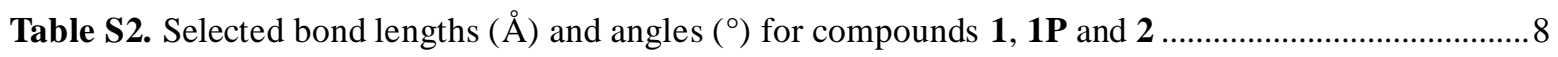

Table S3. Hydrogen bonds of for compounds 1, 1P and $2\left(\AA\right.$ and $\left.^{\circ}\right)$.....................................................13 


\section{Figures}
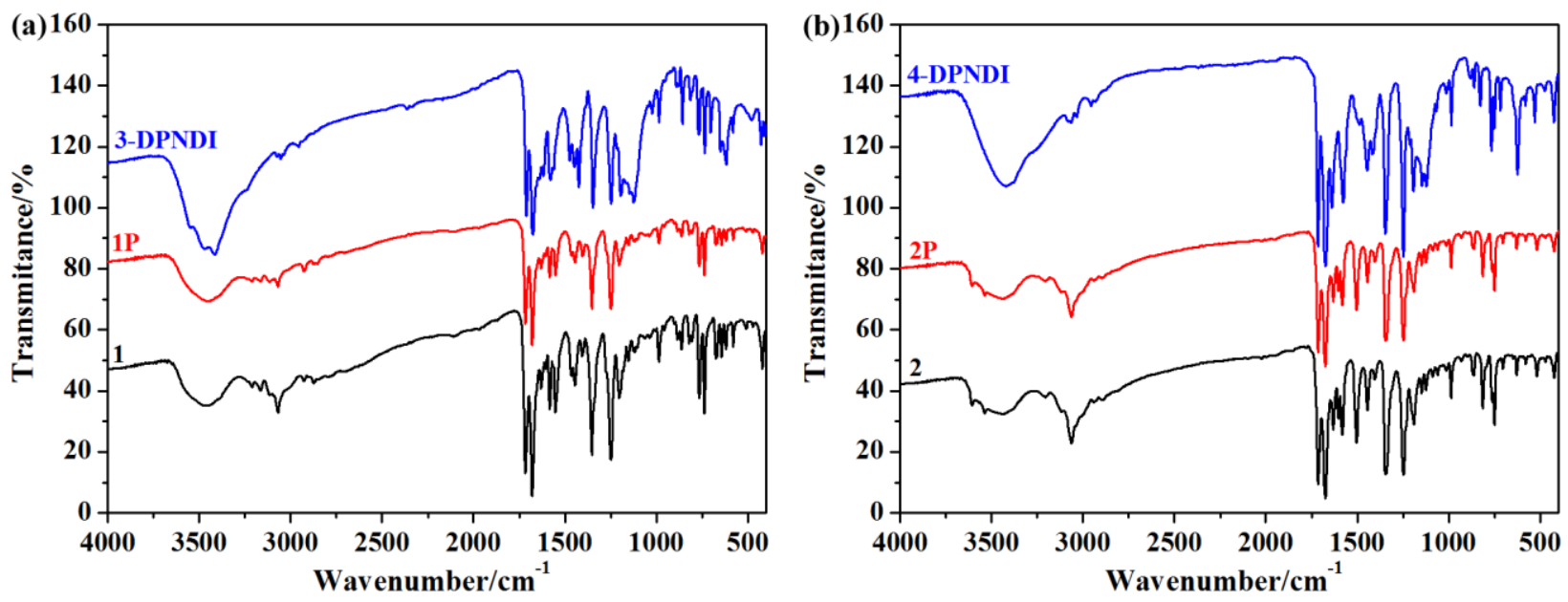

Figure S1. IR spectra of 1-2.

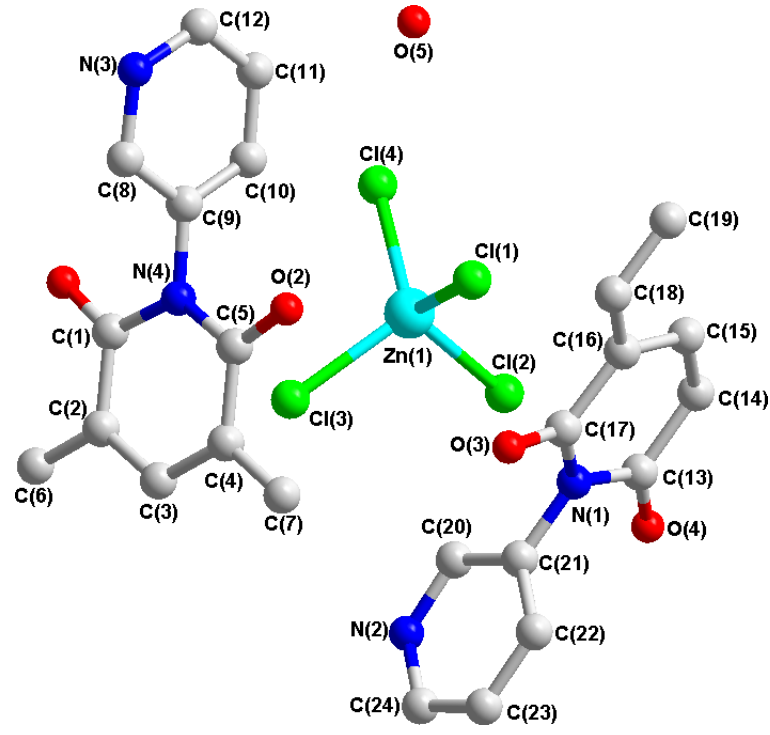

Figure S2. The asymmetric unit of $\mathbf{1}$.

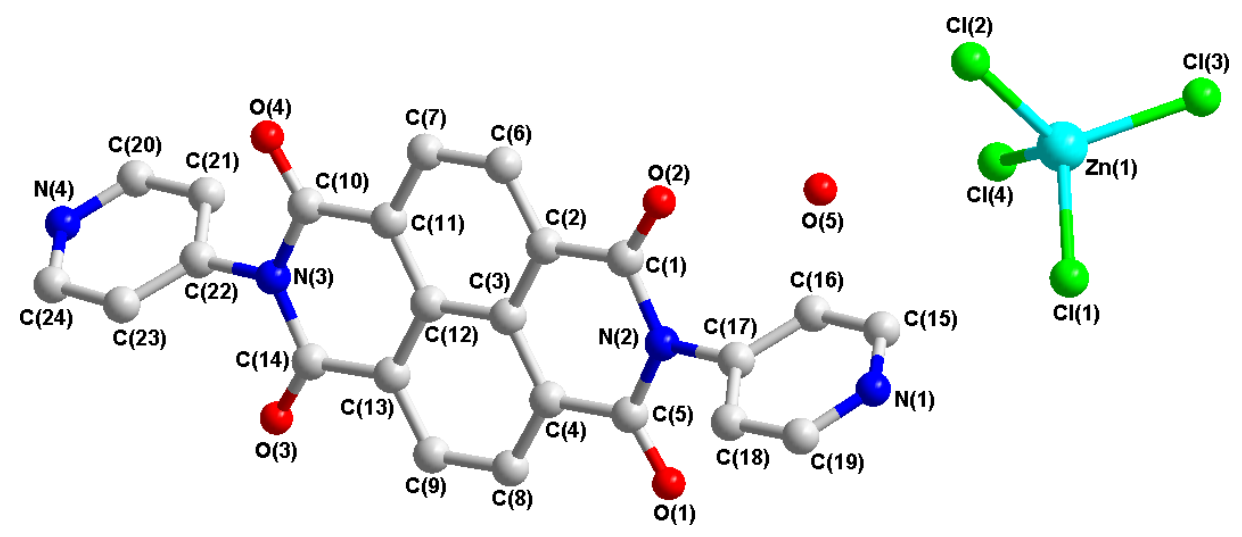

Figure S3. The asymmetric unit of 2. 

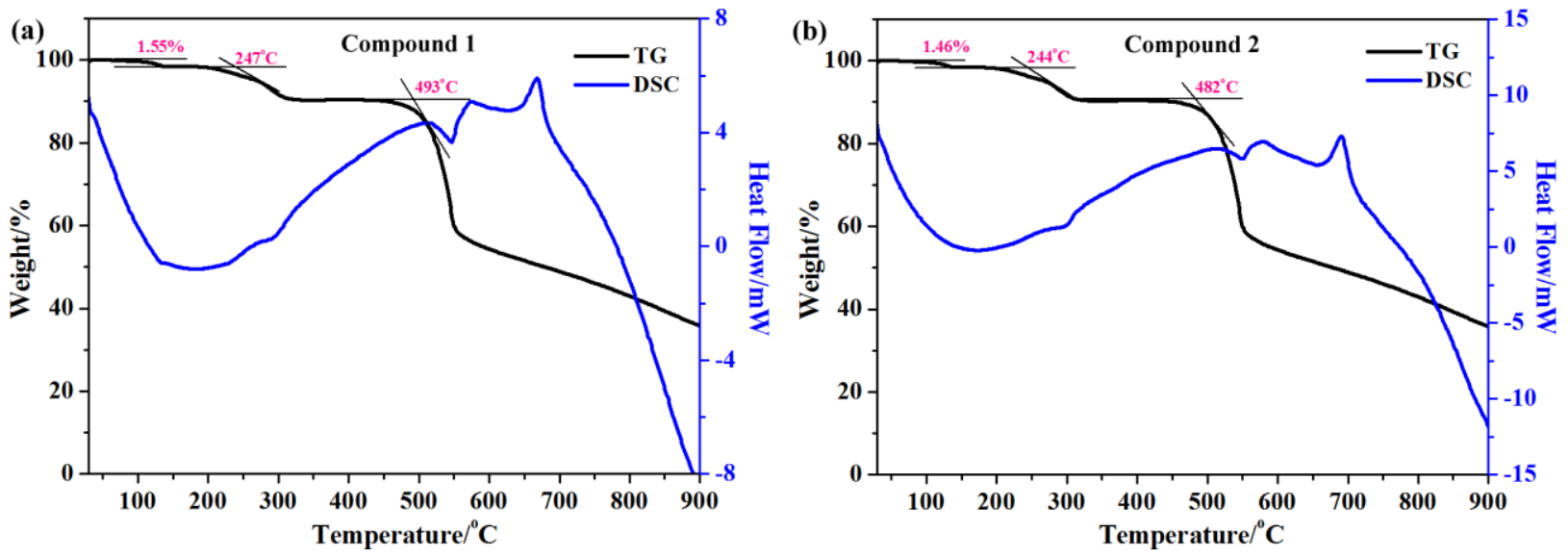

Figure S4. Thermo-gravimetric (TG) and Differential scanning calorimetry (DSC) curves of $\mathbf{1}$ (a) and 2 (b).
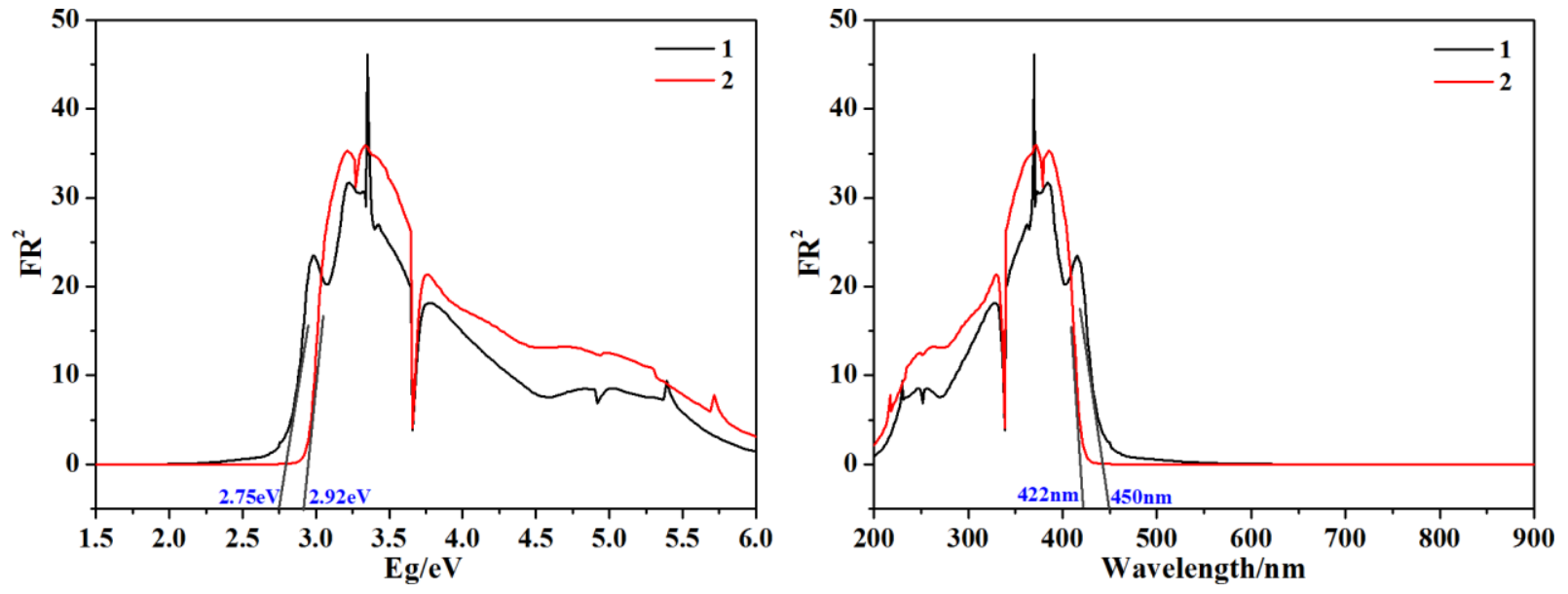

Figure S5. The comparison of energy gaps and UV-vis absorption spectra of $\mathbf{1}$ and $\mathbf{2}$ before irradiation.

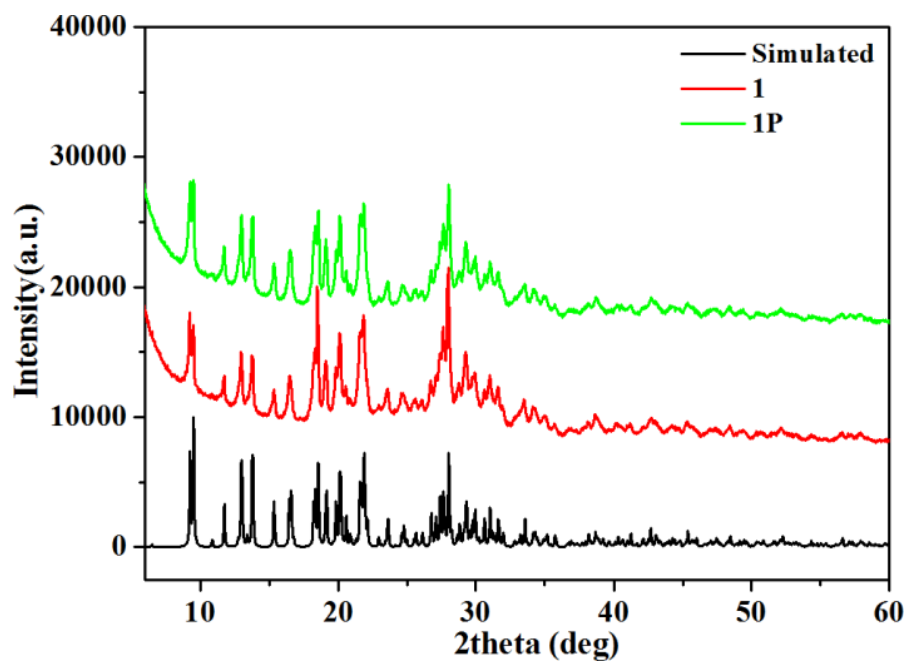

Figure S6. Powder X-ray diffraction (PXRD) patterns for $\mathbf{1}$ and $\mathbf{1 P}$ at room temperature. 


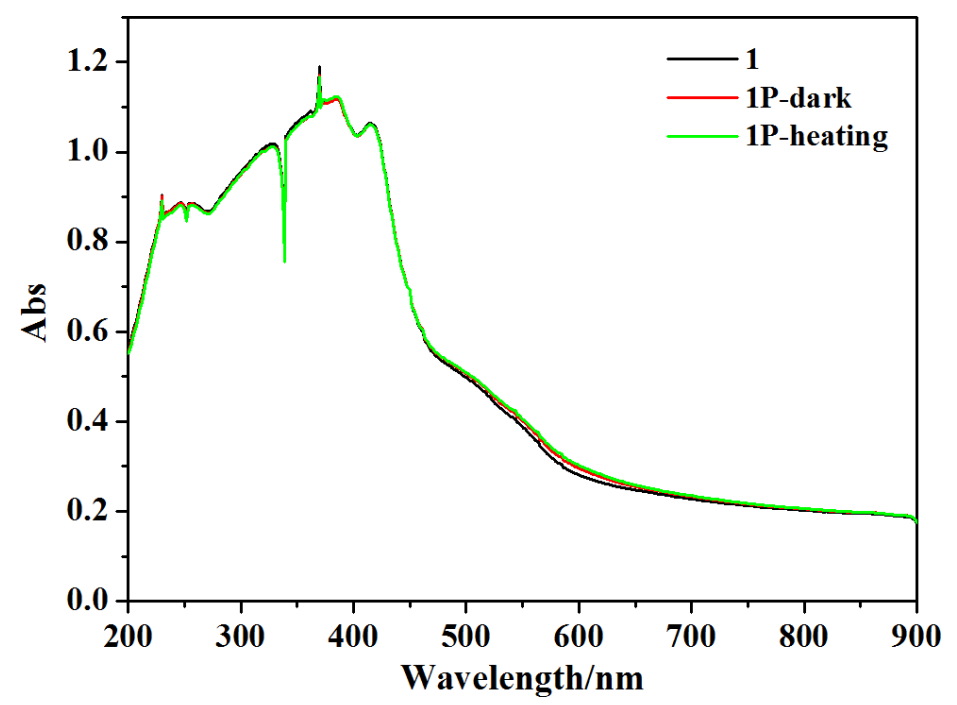

Figure S7. UV-vis absorption spectra of 1, 1P-dark and 1P-heating.

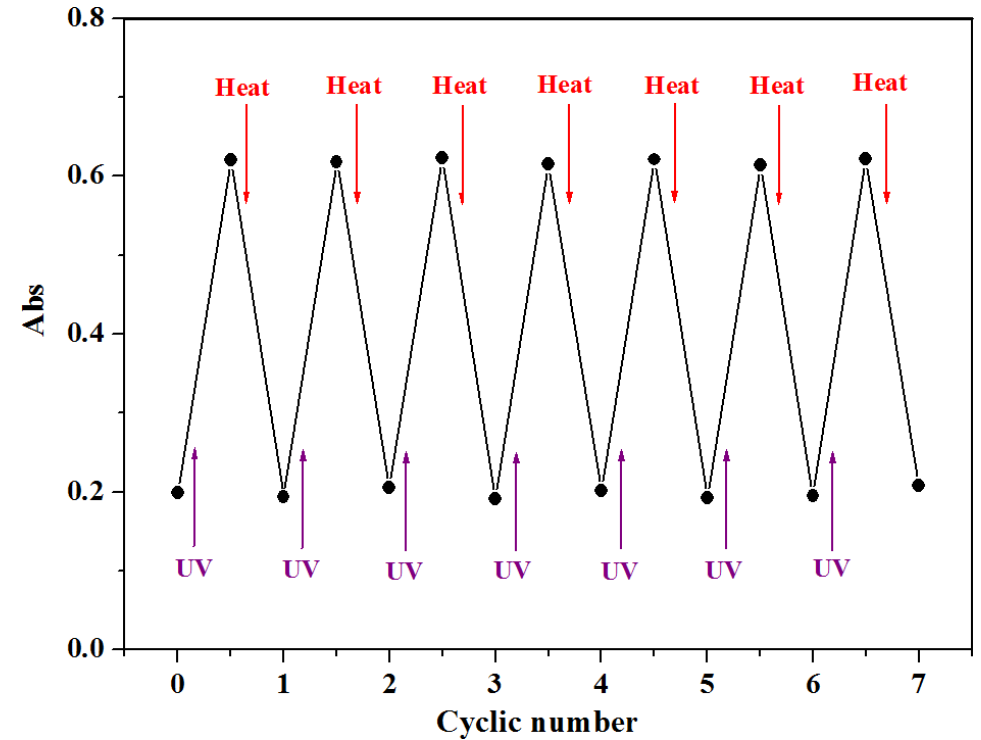

Figure S8. The switching cycles of coloration-decoloration processes of $\mathbf{1}$ upon alternating UV light illumination and thermal treatment 

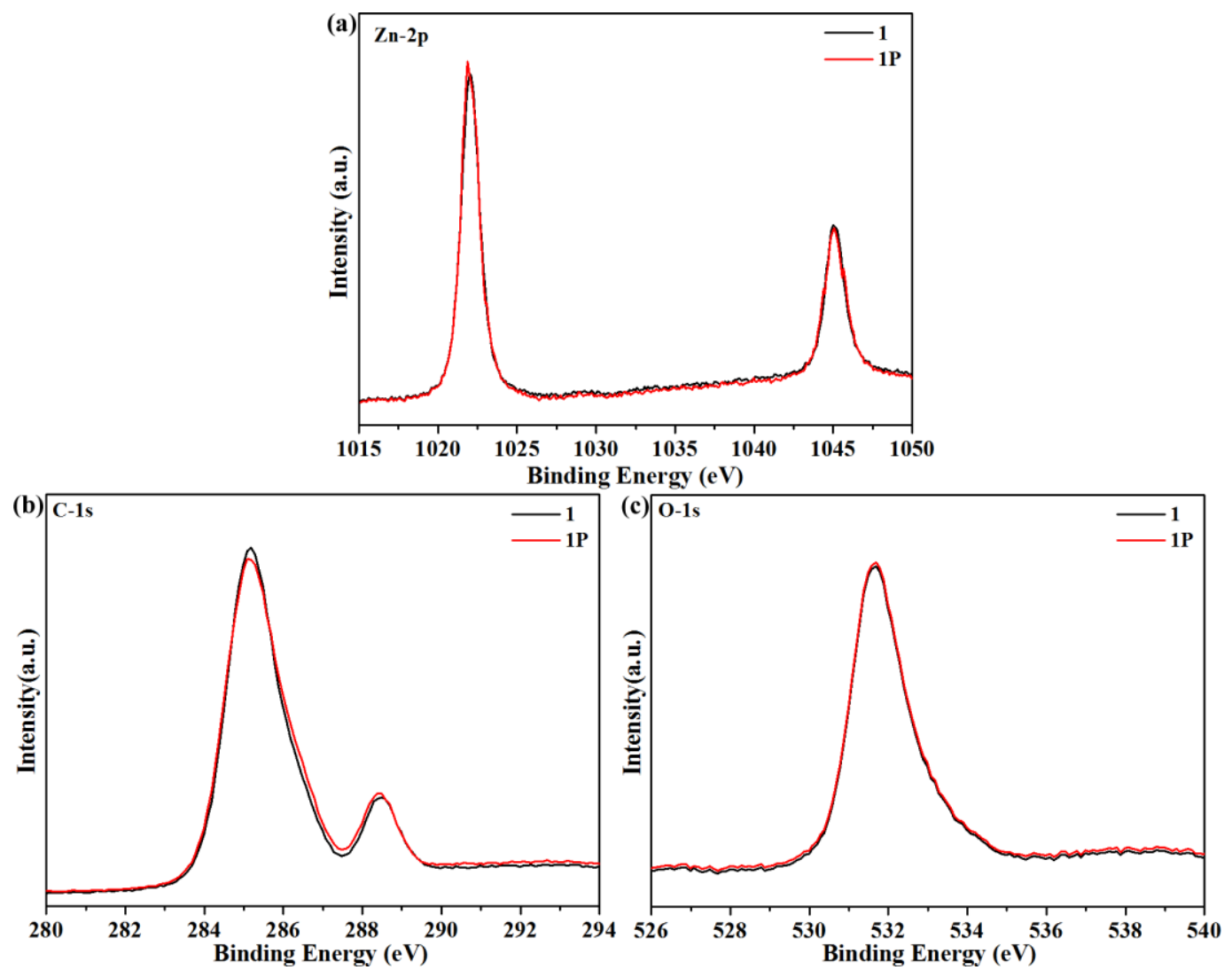

Figure S9. Zn 2p (a), C 1s (b) and O 1s (c) XPS core-level spectra of $\mathbf{1}$ and $\mathbf{1 P}$ at room temperature.

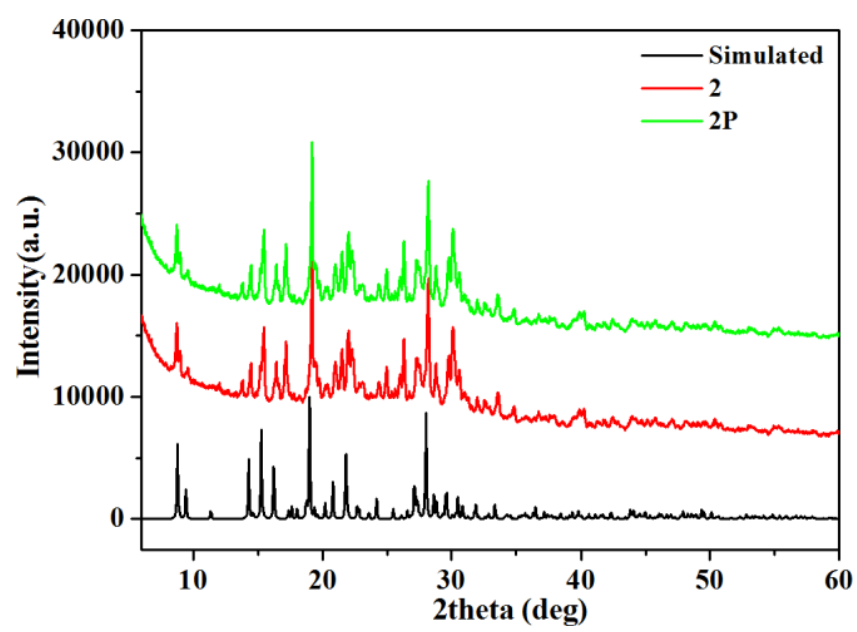

Figure S10. Powder X-ray diffraction (PXRD) patterns for $\mathbf{2}$ and $\mathbf{2 P}$ at room temperature. 

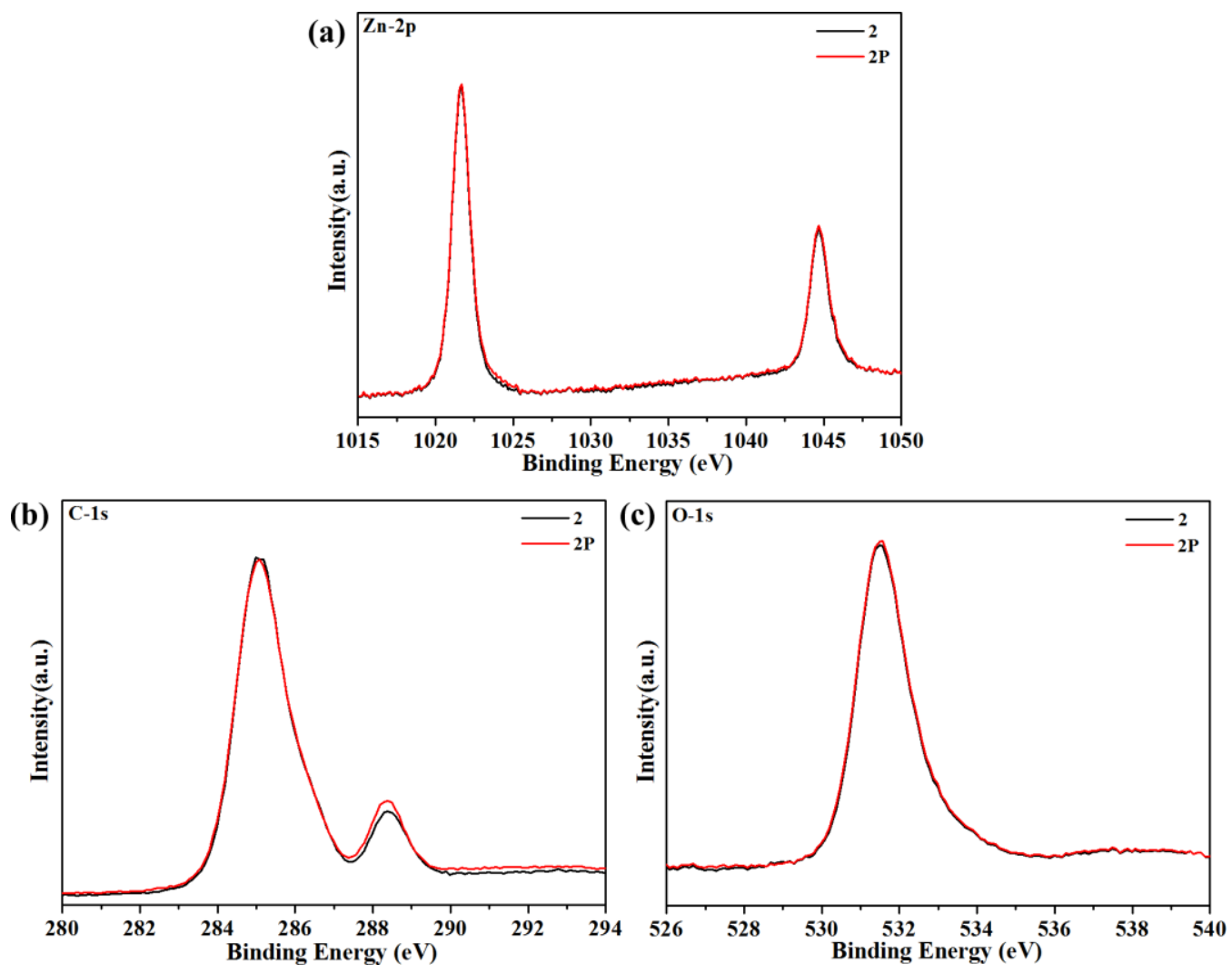

Figure S11. Zn 2p (a), C 1s (b) and O 1s (c) XPS core-level spectra of $\mathbf{2}$ and $\mathbf{2 P}$ at room temperature. 


\section{Tables}

Table S1. Crystallographic data and refinement of compounds 1, 1P and 2

\begin{tabular}{|c|c|c|c|}
\hline Compounds & 1 & $\mathbf{1 P}$ & 2 \\
\hline CCDC code & 1936356 & 1936358 & 1936359 \\
\hline Temperature (K) & $273(2)$ & $273(2)$ & $273(2)$ \\
\hline Empirical formula & $\mathrm{C}_{48} \mathrm{H}_{28} \mathrm{Cl}_{8} \mathrm{~N}_{8} \mathrm{O}_{9} \mathrm{Zn}_{2}$ & $\mathrm{C}_{48} \mathrm{H}_{28} \mathrm{Cl}_{8} \mathrm{~N}_{8} \mathrm{O}_{9} \mathrm{Zn}_{2}$ & $\mathrm{C}_{48} \mathrm{H}_{32} \mathrm{Cl}_{8} \mathrm{~N}_{8} \mathrm{O}_{9} \mathrm{Zn}_{2}$ \\
\hline Formula weight & 1275.12 & 1275.12 & 1279.16 \\
\hline Crystal size (mm) & $0.18 \times 0.17 \times 0.15$ & $0.21 \times 0.19 \times 0.16$ & $0.19 \times 0.09 \times 0.08$ \\
\hline Crystal system & Triclinic & Triclinic & Monoclinic \\
\hline Space group & $P-1$ & $P-1$ & $C c$ \\
\hline$a(\AA)$ & $9.5902(5)$ & $9.5971(11)$ & $19.225(2)$ \\
\hline$b(\AA)$ & $9.6986(5)$ & $9.7026(11)$ & $6.5609(8)$ \\
\hline$c(\AA)$ & $14.1946(7)$ & $14.2077(16)$ & $20.648(3)$ \\
\hline$\alpha\left(^{\circ}\right)$ & $106.2000(10)$ & $106.236(3)$ & 90 \\
\hline$\beta\left(^{\circ}\right)$ & $93.5110(10)$ & $93.543(3)$ & $103.100(3)$ \\
\hline$\gamma\left({ }^{\circ}\right)$ & $93.3620(10)$ & $93.349(3)$ & 90 \\
\hline$V\left(\AA^{3}\right)$ & $1261.50(11)$ & $1263.8(2)$ & $2536.7(5)$ \\
\hline$Z$ & 1 & 1 & 2 \\
\hline $\mathrm{D}_{\mathrm{c}}\left(\mathrm{g} \mathrm{cm}^{-3}\right)$ & 1.678 & 1.675 & 1.675 \\
\hline$F(000)$ & 640 & 640 & 1288 \\
\hline$\mu\left(\mathrm{mm}^{-1}\right)$ & 1.437 & 1.437 & 9.597 \\
\hline$\theta$ range $\left(^{\circ}\right)$ & 2.28 to 28.36 & 2.28 to 28.32 & 3.29 to 28.31 \\
\hline Reflections collected & 18793 & 18458 & 36012 \\
\hline Unique reflections & 6269 & 6248 & 6162 \\
\hline$R_{\text {int }}$ & 0.0229 & 0.0181 & 0.0217 \\
\hline Goodness-of-fit on $F^{2}$ & 1.045 & 1.042 & 1.097 \\
\hline$R_{1} / w R_{2},[I \geq 2 \sigma(I)]^{\mathrm{a}, \mathrm{b}}$ & $0.0459 / 0.1379$ & $0.0441 / 0.1355$ & $0.0305 / 0.0834$ \\
\hline$R_{1} / w R_{2}$, (all data) & $0.0608 / 0.1474$ & $0.0520 / 0.1421$ & $0.0329 / 0.0857$ \\
\hline
\end{tabular}


Table S2. Selected bond lengths ( $(\AA)$ and angles $\left({ }^{\circ}\right)$ for compounds $1, \mathbf{1 P}$ and 2

\begin{tabular}{|c|c|c|c|}
\hline \multicolumn{4}{|c|}{ Compound $\mathbf{1}$} \\
\hline $\mathrm{Zn}(1)-\mathrm{Cl}(1)$ & $2.2300(10)$ & $\mathrm{Zn}(1)-\mathrm{Cl}(3)$ & $2.2456(9)$ \\
\hline $\mathrm{Zn}(1)-\mathrm{Cl}(4)$ & $2.2680(9)$ & $\mathrm{Zn}(1)-\mathrm{Cl}(2)$ & $2.2720(8)$ \\
\hline $\mathrm{C}(1)-\mathrm{O}(1)$ & $1.208(4)$ & $\mathrm{C}(1)-\mathrm{N}(4)$ & $1.402(4)$ \\
\hline$C(1)-C(2)$ & $1.476(4)$ & $C(2)-C(6)$ & $1.373(4)$ \\
\hline$C(2)-C(3)$ & $1.412(4)$ & $C(3)-C(3) \# 1$ & $1.408(5)$ \\
\hline$C(3)-C(4)$ & $1.411(4)$ & $C(4)-C(7)$ & $1.375(4)$ \\
\hline $\mathrm{C}(4)-\mathrm{C}(5)$ & $1.472(4)$ & $\mathrm{C}(5)-\mathrm{O}(2)$ & $1.215(4)$ \\
\hline $\mathrm{C}(5)-\mathrm{N}(4)$ & $1.395(4)$ & $C(6)-C(7) \# 1$ & $1.410(4)$ \\
\hline$C(7)-C(6) \# 1$ & $1.410(4)$ & $\mathrm{C}(8)-\mathrm{C}(9)$ & $1.358(5)$ \\
\hline $\mathrm{C}(8)-\mathrm{N}(3)$ & $1.371(4)$ & $C(9)-C(10)$ & $1.354(4)$ \\
\hline$C(9)-N(4)$ & $1.435(3)$ & $C(10)-C(11)$ & $1.357(4)$ \\
\hline $\mathrm{C}(11)-\mathrm{C}(12)$ & $1.326(5)$ & $C(12)-N(3)$ & $1.330(5)$ \\
\hline $\mathrm{C}(13)-\mathrm{O}(4)$ & $1.206(4)$ & $\mathrm{C}(13)-\mathrm{N}(1)$ & $1.406(4)$ \\
\hline$C(13)-C(14)$ & $1.482(4)$ & $C(14)-C(19) \# 2$ & $1.373(4)$ \\
\hline$C(14)-C(15)$ & $1.410(4)$ & $C(15)-C(16)$ & $1.408(4)$ \\
\hline$C(15)-C(15) \# 2$ & $1.415(6)$ & $C(16)-C(18)$ & $1.373(4)$ \\
\hline$C(16)-C(17)$ & $1.486(4)$ & $\mathrm{C}(17)-\mathrm{O}(3)$ & $1.213(4)$ \\
\hline$C(17)-N(1)$ & $1.397(4)$ & $C(18)-C(19)$ & $1.400(4)$ \\
\hline $\mathrm{C}(19)-\mathrm{C}(14) \# 2$ & $1.373(4)$ & $C(20)-N(2)$ & $1.336(5)$ \\
\hline$C(20)-C(21)$ & $1.369(5)$ & $C(21)-C(22)$ & $1.378(4)$ \\
\hline $\mathrm{C}(21)-\mathrm{N}(1)$ & $1.441(4)$ & $C(22)-C(23)$ & $1.383(4)$ \\
\hline$C(23)-C(24)$ & $1.363(5)$ & $\mathrm{C}(24)-\mathrm{N}(2)$ & $1.326(5)$ \\
\hline $\mathrm{Cl}(1)-\mathrm{Zn}(1)-\mathrm{Cl}(3)$ & $112.89(5)$ & $\mathrm{Cl}(1)-\mathrm{Zn}(1)-\mathrm{Cl}(4)$ & $107.76(4)$ \\
\hline $\mathrm{Cl}(3)-\mathrm{Zn}(1)-\mathrm{Cl}(4)$ & $112.03(4)$ & $\mathrm{Cl}(1)-\mathrm{Zn}(1)-\mathrm{Cl}(2)$ & $109.69(4)$ \\
\hline $\mathrm{Cl}(3)-\mathrm{Zn}(1)-\mathrm{Cl}(2)$ & $104.68(4)$ & $\mathrm{Cl}(4)-\mathrm{Zn}(1)-\mathrm{Cl}(2)$ & $109.76(4)$ \\
\hline $\mathrm{O}(1)-\mathrm{C}(1)-\mathrm{N}(4)$ & $120.2(2)$ & $\mathrm{O}(1)-\mathrm{C}(1)-\mathrm{C}(2)$ & 124.1(3) \\
\hline $\mathrm{N}(4)-\mathrm{C}(1)-\mathrm{C}(2)$ & $115.7(2)$ & $C(6)-C(2)-C(3)$ & $120.8(2)$ \\
\hline$C(6)-C(2)-C(1)$ & 119.2(3) & $\mathrm{C}(3)-\mathrm{C}(2)-\mathrm{C}(1)$ & $120.0(2)$ \\
\hline $\mathrm{C}(3) \# 1-\mathrm{C}(3)-\mathrm{C}(4)$ & 119.1(3) & $C(3) \# 1-C(3)-C(2)$ & 119.3(3) \\
\hline$C(4)-C(3)-C(2)$ & $121.6(2)$ & $C(7)-C(4)-C(3)$ & $120.8(2)$ \\
\hline$C(7)-C(4)-C(5)$ & $119.6(2)$ & $C(3)-C(4)-C(5)$ & $119.6(3)$ \\
\hline $\mathrm{O}(2)-\mathrm{C}(5)-\mathrm{N}(4)$ & $120.1(2)$ & $\mathrm{O}(2)-\mathrm{C}(5)-\mathrm{C}(4)$ & $123.4(3)$ \\
\hline $\mathrm{N}(4)-\mathrm{C}(5)-\mathrm{C}(4)$ & $116.5(2)$ & $C(2)-C(6)-C(7) \# 1$ & $120.0(3)$ \\
\hline $\mathrm{C}(4)-\mathrm{C}(7)-\mathrm{C}(6) \# 1$ & $120.0(3)$ & $\mathrm{C}(9)-\mathrm{C}(8)-\mathrm{N}(3)$ & $119.3(3)$ \\
\hline
\end{tabular}




\begin{tabular}{|c|c|c|c|}
\hline $\mathrm{C}(10)-\mathrm{C}(9)-\mathrm{C}(8)$ & 119.1(3) & $\mathrm{C}(10)-\mathrm{C}(9)-\mathrm{N}(4)$ & 121.1(3) \\
\hline $\mathrm{C}(8)-\mathrm{C}(9)-\mathrm{N}(4)$ & $119.8(3)$ & $C(9)-C(10)-C(11)$ & $119.6(3)$ \\
\hline$C(12)-C(11)-C(10)$ & $121.4(3)$ & $\mathrm{C}(11)-\mathrm{C}(12)-\mathrm{N}(3)$ & $119.6(3)$ \\
\hline $\mathrm{C}(12)-\mathrm{C}(11)-\mathrm{C}(10)$ & $121.4(3)$ & $\mathrm{C}(11)-\mathrm{C}(12)-\mathrm{N}(3)$ & $119.6(3)$ \\
\hline $\mathrm{O}(4)-\mathrm{C}(13)-\mathrm{N}(1)$ & $120.5(3)$ & $\mathrm{O}(4)-\mathrm{C}(13)-\mathrm{C}(14)$ & $123.5(3)$ \\
\hline $\mathrm{N}(1)-\mathrm{C}(13)-\mathrm{C}(14)$ & $116.0(3)$ & $C(19) \# 2-C(14)-(15)$ & $120.1(3)$ \\
\hline $\mathrm{C}(19) \# 2-\mathrm{C}(14)-\mathrm{C}(13)$ & $119.9(3)$ & $\mathrm{C}(15)-\mathrm{C}(14)-\mathrm{C}(13)$ & $120.0(3)$ \\
\hline$C(16)-C(15)-C(14)$ & $121.5(3)$ & $C(16)-C(15)-(15) \# 2$ & $118.8(3)$ \\
\hline $\mathrm{C}(14)-\mathrm{C}(15)-\mathrm{C}(15) \# 2$ & $119.6(3)$ & $C(18)-C(16)-C(15)$ & $120.6(3)$ \\
\hline$C(18)-C(16)-C(17)$ & $119.5(3)$ & $C(15)-C(16)-C(17)$ & $119.9(3)$ \\
\hline $\mathrm{O}(3)-\mathrm{C}(17)-\mathrm{N}(1)$ & $120.5(3)$ & $\mathrm{O}(3)-\mathrm{C}(17)-\mathrm{C}(16)$ & $123.3(3)$ \\
\hline $\mathrm{N}(1)-\mathrm{C}(17)-\mathrm{C}(16)$ & $116.2(3)$ & $C(16)-C(18)-C(19)$ & $120.4(3)$ \\
\hline$C(14) \# 2-C(19)-C(18)$ & $120.5(3)$ & $\mathrm{N}(2)-\mathrm{C}(20)-\mathrm{C}(21)$ & $118.3(3)$ \\
\hline$C(20)-C(21)-C(22)$ & $119.9(3)$ & $\mathrm{C}(20)-\mathrm{C}(21)-\mathrm{N}(1)$ & $118.8(3)$ \\
\hline $\mathrm{C}(22)-\mathrm{C}(21)-\mathrm{N}(1)$ & $121.3(3)$ & $\mathrm{C}(21)-\mathrm{C}(22)-\mathrm{C}(23)$ & $119.5(3)$ \\
\hline $\mathrm{C}(24)-\mathrm{C}(23)-\mathrm{C}(22)$ & $118.9(3)$ & $\mathrm{N}(2)-\mathrm{C}(24)-\mathrm{C}(23)$ & $119.7(3)$ \\
\hline $\mathrm{C}(17)-\mathrm{N}(1)-\mathrm{C}(13)$ & $126.1(2)$ & $\mathrm{C}(17)-\mathrm{N}(1)-\mathrm{C}(21)$ & $117.8(3)$ \\
\hline $\mathrm{C}(13)-\mathrm{N}(1)-\mathrm{C}(21)$ & $115.8(3)$ & $\mathrm{C}(24)-\mathrm{N}(2)-\mathrm{C}(20)$ & $123.6(3)$ \\
\hline $\mathrm{C}(12)-\mathrm{N}(3)-\mathrm{C}(8)$ & $120.8(3)$ & $\mathrm{C}(5)-\mathrm{N}(4)-\mathrm{C}(1)$ & $126.4(2)$ \\
\hline $\mathrm{C}(5)-\mathrm{N}(4)-\mathrm{C}(9)$ & $117.0(2)$ & $\mathrm{C}(1)-\mathrm{N}(4)-\mathrm{C}(9)$ & $116.3(2)$ \\
\hline \multicolumn{4}{|c|}{$\# 1-x+1,-y+1,-z ; \# 2-x+2,-y,-z+1$} \\
\hline \multicolumn{4}{|c|}{ Compound 1P } \\
\hline $\mathrm{Zn}(1)-\mathrm{Cl}(1)$ & $2.2320(9)$ & $\mathrm{Zn}(1)-\mathrm{Cl}(3)$ & $2.2471(9)$ \\
\hline $\mathrm{Zn}(1)-\mathrm{Cl}(4)$ & $2.2657(8)$ & $\mathrm{Zn}(1)-\mathrm{Cl}(2)$ & $2.2707(8)$ \\
\hline $\mathrm{C}(1)-\mathrm{O}(1)$ & $1.207(3)$ & $\mathrm{C}(1)-\mathrm{N}(4)$ & $1.402(3)$ \\
\hline $\mathrm{C}(1)-\mathrm{C}(2)$ & $1.478(3)$ & $C(2)-C(6)$ & $1.376(3)$ \\
\hline$C(2)-C(3)$ & $1.410(3)$ & $\mathrm{C}(3)-\mathrm{C}(4)$ & $1.412(3)$ \\
\hline $\mathrm{C}(3)-\mathrm{C}(3) \# 1$ & $1.412(4)$ & $\mathrm{C}(4)-\mathrm{C}(7)$ & $1.371(4)$ \\
\hline $\mathrm{C}(4)-\mathrm{C}(5)$ & $1.474(3)$ & $\mathrm{C}(5)-\mathrm{O}(2)$ & $1.215(3)$ \\
\hline $\mathrm{C}(5)-\mathrm{N}(4)$ & $1.394(3)$ & $\mathrm{C}(6)-\mathrm{C}(7) \# 1$ & $1.409(3)$ \\
\hline$C(7)-C(6) \# 1$ & $1.409(3)$ & $\mathrm{C}(8)-\mathrm{C}(9)$ & $1.362(4)$ \\
\hline $\mathrm{C}(8)-\mathrm{N}(3)$ & $1.366(4)$ & $\mathrm{C}(9)-\mathrm{C}(10)$ & $1.355(4)$ \\
\hline $\mathrm{C}(9)-\mathrm{N}(4)$ & $1.434(3)$ & $\mathrm{C}(10)-\mathrm{C}(11)$ & $1.359(4)$ \\
\hline $\mathrm{C}(11)-\mathrm{C}(12)$ & $1.328(5)$ & $\mathrm{C}(12)-\mathrm{N}(3)$ & $1.328(5)$ \\
\hline $\mathrm{C}(13)-\mathrm{O}(4)$ & $1.205(4)$ & $\mathrm{C}(13)-\mathrm{N}(1)$ & $1.406(4)$ \\
\hline$C(13)-C(14)$ & $1.483(4)$ & $C(14)-C(19) \# 2$ & $1.375(4)$ \\
\hline$C(14)-C(15)$ & $1.410(4)$ & $\mathrm{C}(15)-\mathrm{C}(15) \# 2$ & $1.411(5)$ \\
\hline
\end{tabular}




\begin{tabular}{|c|c|c|c|}
\hline $\mathrm{C}(15)-\mathrm{C}(16)$ & $1.413(4)$ & $\mathrm{C}(16)-\mathrm{C}(18)$ & $1.368(4)$ \\
\hline$C(16)-C(17)$ & $1.481(4)$ & $\mathrm{C}(17)-\mathrm{O}(3)$ & $1.216(4)$ \\
\hline $\mathrm{C}(17)-\mathrm{N}(1)$ & $1.395(4)$ & $\mathrm{C}(18)-\mathrm{C}(19)$ & $1.401(4)$ \\
\hline$C(19)-C(14) \# 2$ & $1.375(4)$ & $\mathrm{C}(20)-\mathrm{N}(2)$ & $1.336(4)$ \\
\hline$C(20)-C(21)$ & $1.369(4)$ & $C(21)-C(22)$ & $1.380(4)$ \\
\hline$C(21)-N(1)$ & $1.440(3)$ & $\mathrm{C}(22)-\mathrm{C}(23)$ & $1.387(4)$ \\
\hline$C(23)-C(24)$ & $1.364(5)$ & $\mathrm{C}(24)-\mathrm{N}(2)$ & $1.325(5)$ \\
\hline $\mathrm{Cl}(1)-\mathrm{Zn}(1)-\mathrm{Cl}(3)$ & $112.85(4)$ & $\mathrm{Cl}(1)-\mathrm{Zn}(1)-\mathrm{Cl}(4)$ & $107.79(4)$ \\
\hline $\mathrm{Cl}(3)-\mathrm{Zn}(1)-\mathrm{Cl}(4)$ & $111.85(4)$ & $\mathrm{Cl}(1)-\mathrm{Zn}(1)-\mathrm{Cl}(2)$ & $109.72(3)$ \\
\hline $\mathrm{Cl}(3)-\mathrm{Zn}(1)-\mathrm{Cl}(2)$ & $104.82(3)$ & $\mathrm{Cl}(4)-\mathrm{Zn}(1)-\mathrm{Cl}(2)$ & $109.78(4)$ \\
\hline $\mathrm{O}(1)-\mathrm{C}(1)-\mathrm{N}(4)$ & $120.3(2)$ & $\mathrm{O}(1)-\mathrm{C}(1)-\mathrm{C}(2)$ & $123.9(2)$ \\
\hline $\mathrm{N}(4)-\mathrm{C}(1)-\mathrm{C}(2)$ & $115.8(2)$ & $\mathrm{C}(6)-\mathrm{C}(2)-\mathrm{C}(3)$ & $121.0(2)$ \\
\hline$C(6)-C(2)-C(1)$ & $119.2(2)$ & $\mathrm{C}(3)-\mathrm{C}(2)-\mathrm{C}(1)$ & $119.8(2)$ \\
\hline$C(2)-C(3)-C(4)$ & $122.1(2)$ & $\mathrm{C}(2)-\mathrm{C}(3)-\mathrm{C}(3) \# 1$ & $119.2(3)$ \\
\hline $\mathrm{C}(4)-\mathrm{C}(3)-\mathrm{C}(3) \# 1$ & $118.8(3)$ & $C(7)-C(4)-C(3)$ & $121.2(2)$ \\
\hline$C(7)-C(4)-C(5)$ & $119.7(2)$ & $C(3)-C(4)-C(5)$ & 119.1(2) \\
\hline $\mathrm{O}(2)-\mathrm{C}(5)-\mathrm{N}(4)$ & $120.0(2)$ & $\mathrm{O}(2)-\mathrm{C}(5)-\mathrm{C}(4)$ & $123.2(2)$ \\
\hline $\mathrm{N}(4)-\mathrm{C}(5)-\mathrm{C}(4)$ & $116.7(2)$ & $\mathrm{C}(2)-\mathrm{C}(6)-\mathrm{C}(7) \# 1$ & $119.8(2)$ \\
\hline $\mathrm{C}(4)-\mathrm{C}(7)-\mathrm{C}(6) \# 1$ & $120.1(2)$ & $\mathrm{C}(9)-\mathrm{C}(8)-\mathrm{N}(3)$ & $119.4(3)$ \\
\hline $\mathrm{C}(10)-\mathrm{C}(9)-\mathrm{C}(8)$ & $119.0(2)$ & $\mathrm{C}(10)-\mathrm{C}(9)-\mathrm{N}(4)$ & $121.2(2)$ \\
\hline $\mathrm{C}(8)-\mathrm{C}(9)-\mathrm{N}(4)$ & $119.8(2)$ & $\mathrm{C}(9)-\mathrm{C}(10)-\mathrm{C}(11)$ & $119.5(3)$ \\
\hline$C(12)-C(11)-C(10)$ & $121.4(3)$ & $\mathrm{N}(3)-\mathrm{C}(12)-\mathrm{C}(11)$ & $119.5(3)$ \\
\hline $\mathrm{O}(4)-\mathrm{C}(13)-\mathrm{N}(1)$ & $120.5(3)$ & $\mathrm{O}(4)-\mathrm{C}(13)-\mathrm{C}(14)$ & $123.7(3)$ \\
\hline $\mathrm{N}(1)-\mathrm{C}(13)-\mathrm{C}(14)$ & $115.8(3)$ & $\mathrm{C}(19) \# 2-\mathrm{C}(14)-(15)$ & $119.9(3)$ \\
\hline $\mathrm{C}(19) \# 2-\mathrm{C}(14)-\mathrm{C}(13)$ & $119.8(3)$ & $\mathrm{C}(15)-\mathrm{C}(14)-\mathrm{C}(13)$ & $120.3(3)$ \\
\hline $\mathrm{C}(14)-\mathrm{C}(15)-\mathrm{C}(15) \# 2$ & $119.7(3)$ & $C(14)-C(15)-C(16)$ & $121.2(2)$ \\
\hline $\mathrm{C}(15) \# 2-\mathrm{C}(15)-\mathrm{C}(16)$ & 119.1(3) & $C(18)-C(16)-C(15)$ & $120.1(3)$ \\
\hline $\mathrm{C}(18)-\mathrm{C}(16)-\mathrm{C}(17)$ & $120.0(3)$ & $C(15)-C(16)-C(17)$ & $119.9(3)$ \\
\hline $\mathrm{O}(3)-\mathrm{C}(17)-\mathrm{N}(1)$ & $120.4(3)$ & $\mathrm{O}(3)-\mathrm{C}(17)-\mathrm{C}(16)$ & $123.1(3)$ \\
\hline $\mathrm{N}(1)-\mathrm{C}(17)-\mathrm{C}(16)$ & $116.5(2)$ & $C(16)-C(18)-C(19)$ & $120.9(3)$ \\
\hline $\mathrm{C}(14) \# 2-\mathrm{C}(19)-\mathrm{C}(18)$ & $120.3(3)$ & $N(2)-C(20)-C(21)$ & $118.5(3)$ \\
\hline $\mathrm{C}(20)-\mathrm{C}(21)-\mathrm{C}(22)$ & $119.7(3)$ & $\mathrm{C}(20)-\mathrm{C}(21)-\mathrm{N}(1)$ & $119.0(3)$ \\
\hline $\mathrm{C}(22)-\mathrm{C}(21)-\mathrm{N}(1)$ & 121.2(3) & $\mathrm{C}(21)-\mathrm{C}(22)-\mathrm{C}(23)$ & $119.5(3)$ \\
\hline $\mathrm{C}(24)-\mathrm{C}(23)-\mathrm{C}(22)$ & $118.9(3)$ & $\mathrm{N}(2)-\mathrm{C}(24)-\mathrm{C}(23)$ & $119.7(3)$ \\
\hline $\mathrm{C}(17)-\mathrm{N}(1)-\mathrm{C}(13)$ & $126.1(2)$ & $\mathrm{C}(17)-\mathrm{N}(1)-\mathrm{C}(21)$ & $117.8(2)$ \\
\hline $\mathrm{C}(13)-\mathrm{N}(1)-\mathrm{C}(21)$ & $115.9(2)$ & $\mathrm{C}(24)-\mathrm{N}(2)-\mathrm{C}(20)$ & $123.6(3)$ \\
\hline
\end{tabular}




\begin{tabular}{|c|c|c|c|}
\hline $\mathrm{C}(12)-\mathrm{N}(3)-\mathrm{C}(8)$ & $121.0(3)$ & $\mathrm{C}(5)-\mathrm{N}(4)-\mathrm{C}(1)$ & $126.39(19)$ \\
\hline $\mathrm{C}(5)-\mathrm{N}(4)-\mathrm{C}(9)$ & $117.0(2)$ & $\mathrm{C}(1)-\mathrm{N}(4)-\mathrm{C}(9)$ & $116.3(2)$ \\
\hline \multicolumn{4}{|c|}{$\# 1-x+1,-y+1,-z ; \# 2-x+2,-y,-z+1$} \\
\hline \multicolumn{4}{|c|}{ Compound 2} \\
\hline $\mathrm{Zn}(1)-\mathrm{Cl}(4)$ & $2.2477(8)$ & $\mathrm{Zn}(1)-\mathrm{Cl}(3)$ & $2.2581(8)$ \\
\hline $\mathrm{Zn}(1)-\mathrm{Cl}(2)$ & $2.2664(8)$ & $\mathrm{Zn}(1)-\mathrm{Cl}(1)$ & $2.2935(7)$ \\
\hline $\mathrm{C}(1)-\mathrm{O}(2)$ & $1.202(3)$ & $\mathrm{C}(1)-\mathrm{N}(2)$ & $1.410(3)$ \\
\hline$C(1)-C(2)$ & $1.478(3)$ & $C(2)-C(6)$ & $1.379(3)$ \\
\hline$C(2)-C(3)$ & $1.410(3)$ & $\mathrm{C}(3)-\mathrm{C}(4)$ & $1.407(3)$ \\
\hline $\mathrm{C}(3)-\mathrm{C}(12)$ & $1.413(3)$ & $C(4)-C(8)$ & $1.384(4)$ \\
\hline$C(4)-C(5)$ & $1.474(3)$ & $\mathrm{C}(5)-\mathrm{O}(1)$ & $1.206(3)$ \\
\hline $\mathrm{C}(5)-\mathrm{N}(2)$ & $1.402(3)$ & $\mathrm{C}(6)-\mathrm{C}(7)$ & $1.399(4)$ \\
\hline $\mathrm{C}(7)-\mathrm{C}(11)$ & $1.377(4)$ & $\mathrm{C}(8)-\mathrm{C}(9)$ & $1.403(4)$ \\
\hline $\mathrm{C}(9)-\mathrm{C}(13)$ & $1.362(3)$ & $\mathrm{C}(10)-\mathrm{O}(4)$ & $1.207(4)$ \\
\hline$C(10)-N(3)$ & $1.404(3)$ & $C(10)-C(11)$ & $1.475(3)$ \\
\hline $\mathrm{C}(11)-\mathrm{C}(12)$ & $1.409(3)$ & $C(12)-C(13)$ & $1.411(3)$ \\
\hline$C(13)-C(14)$ & $1.484(3)$ & $\mathrm{C}(14)-\mathrm{O}(3)$ & $1.207(3)$ \\
\hline $\mathrm{C}(14)-\mathrm{N}(3)$ & $1.407(3)$ & $\mathrm{C}(15)-\mathrm{N}(1)$ & $1.320(5)$ \\
\hline$C(15)-C(16)$ & $1.379(4)$ & $C(16)-C(17)$ & $1.376(4)$ \\
\hline $\mathrm{C}(17)-\mathrm{C}(18)$ & $1.380(4)$ & $\mathrm{C}(17)-\mathrm{N}(2)$ & $1.442(3)$ \\
\hline $\mathrm{C}(18)-\mathrm{C}(19)$ & $1.376(4)$ & $\mathrm{C}(19)-\mathrm{N}(1)$ & $1.331(4)$ \\
\hline $\mathrm{C}(20)-\mathrm{N}(4)$ & $1.316(6)$ & $C(20)-C(21)$ & $1.362(5)$ \\
\hline$C(21)-C(22)$ & $1.401(4)$ & $C(22)-C(23)$ & $1.363(5)$ \\
\hline $\mathrm{C}(22)-\mathrm{N}(3)$ & $1.438(3)$ & $C(23)-C(24)$ & $1.399(5)$ \\
\hline $\mathrm{C}(24)-\mathrm{N}(4)$ & $1.321(6)$ & & \\
\hline $\mathrm{Cl}(4)-\mathrm{Zn}(1)-\mathrm{Cl}(3)$ & $111.90(3)$ & $\mathrm{Cl}(4)-\mathrm{Zn}(1)-\mathrm{Cl}(2)$ & $105.76(3)$ \\
\hline $\mathrm{Cl}(3)-\mathrm{Zn}(1)-\mathrm{Cl}(2)$ & $115.49(4)$ & $\mathrm{Cl}(4)-\mathrm{Zn}(1)-\mathrm{Cl}(1)$ & $111.40(3)$ \\
\hline $\mathrm{Cl}(3)-\mathrm{Zn}(1)-\mathrm{Cl}(1)$ & $106.78(3)$ & $\mathrm{Cl}(2)-\mathrm{Zn}(1)-\mathrm{Cl}(1)$ & $105.39(3)$ \\
\hline $\mathrm{O}(2)-\mathrm{C}(1)-\mathrm{N}(2)$ & $120.4(2)$ & $\mathrm{O}(2)-\mathrm{C}(1)-\mathrm{C}(2)$ & $124.0(2)$ \\
\hline $\mathrm{N}(2)-\mathrm{C}(1)-\mathrm{C}(2)$ & $115.7(2)$ & $C(6)-C(2)-C(3)$ & $120.5(2)$ \\
\hline$C(6)-C(2)-C(1)$ & $119.3(2)$ & $C(3)-C(2)-C(1)$ & $120.2(2)$ \\
\hline $\mathrm{C}(4)-\mathrm{C}(3)-\mathrm{C}(2)$ & $121.8(2)$ & $\mathrm{C}(4)-\mathrm{C}(3)-\mathrm{C}(12)$ & $119.0(2)$ \\
\hline $\mathrm{C}(2)-\mathrm{C}(3)-\mathrm{C}(12)$ & $119.3(2)$ & $\mathrm{C}(8)-\mathrm{C}(4)-\mathrm{C}(3)$ & $120.7(2)$ \\
\hline$C(8)-C(4)-C(5)$ & $119.5(2)$ & $\mathrm{C}(3)-\mathrm{C}(4)-\mathrm{C}(5)$ & $119.8(2)$ \\
\hline $\mathrm{O}(1)-\mathrm{C}(5)-\mathrm{N}(2)$ & $120.6(2)$ & $\mathrm{O}(1)-\mathrm{C}(5)-\mathrm{C}(4)$ & $122.9(2)$ \\
\hline $\mathrm{N}(2)-\mathrm{C}(5)-\mathrm{C}(4)$ & $116.4(2)$ & $C(2)-C(6)-C(7)$ & $120.5(2)$ \\
\hline
\end{tabular}




\begin{tabular}{llll}
\hline $\mathrm{C}(11)-\mathrm{C}(7)-\mathrm{C}(6)$ & $119.6(2)$ & $\mathrm{C}(4)-\mathrm{C}(8)-\mathrm{C}(9)$ & $119.8(2)$ \\
$\mathrm{C}(13)-\mathrm{C}(9)-\mathrm{C}(8)$ & $120.4(2)$ & $\mathrm{O}(4)-\mathrm{C}(10)-\mathrm{N}(3)$ & $120.0(2)$ \\
$\mathrm{O}(4)-\mathrm{C}(10)-\mathrm{C}(11)$ & $123.4(3)$ & $\mathrm{N}(3)-\mathrm{C}(10)-\mathrm{C}(11)$ & $116.6(2)$ \\
$\mathrm{C}(7)-\mathrm{C}(11)-\mathrm{C}(12)$ & $121.3(2)$ & $\mathrm{C}(7)-\mathrm{C}(11)-\mathrm{C}(10)$ & $119.1(2)$ \\
$\mathrm{C}(12)-\mathrm{C}(11)-\mathrm{C}(10)$ & $119.6(2)$ & $\mathrm{C}(11)-\mathrm{C}(12)-\mathrm{C}(13)$ & $122.2(2)$ \\
$\mathrm{C}(11)-\mathrm{C}(12)-\mathrm{C}(3)$ & $118.7(2)$ & $\mathrm{C}(13)-\mathrm{C}(12)-\mathrm{C}(3)$ & $119.1(2)$ \\
$\mathrm{C}(9)-\mathrm{C}(13)-\mathrm{C}(12)$ & $121.0(2)$ & $\mathrm{C}(9)-\mathrm{C}(13)-\mathrm{C}(14)$ & $119.5(2)$ \\
$\mathrm{C}(12)-\mathrm{C}(13)-\mathrm{C}(14)$ & $119.5(2)$ & $\mathrm{O}(3)-\mathrm{C}(14)-\mathrm{N}(3)$ & $120.3(2)$ \\
$\mathrm{O}(3)-\mathrm{C}(14)-\mathrm{C}(13)$ & $123.5(2)$ & $\mathrm{N}(3)-\mathrm{C}(14)-\mathrm{C}(13)$ & $116.2(2)$ \\
$\mathrm{N}(1)-\mathrm{C}(15)-\mathrm{C}(16)$ & $120.1(3)$ & $\mathrm{C}(17)-\mathrm{C}(16)-\mathrm{C}(15)$ & $118.3(3)$ \\
$\mathrm{C}(16)-\mathrm{C}(17)-\mathrm{C}(18)$ & $120.5(2)$ & $\mathrm{C}(16)-\mathrm{C}(17)-\mathrm{N}(2)$ & $119.8(2)$ \\
$\mathrm{C}(18)-\mathrm{C}(17)-\mathrm{N}(2)$ & $119.7(2)$ & $\mathrm{C}(19)-\mathrm{C}(18)-\mathrm{C}(17)$ & $118.7(3)$ \\
$\mathrm{N}(1)-\mathrm{C}(19)-\mathrm{C}(18)$ & $119.5(3)$ & $\mathrm{N}(4)-\mathrm{C}(20)-\mathrm{C}(21)$ & $119.9(4)$ \\
$\mathrm{C}(20)-\mathrm{C}(21)-\mathrm{C}(22)$ & $118.1(4)$ & $\mathrm{C}(23)-\mathrm{C}(22)-\mathrm{C}(21)$ & $121.2(3)$ \\
$\mathrm{C}(23)-\mathrm{C}(22)-\mathrm{N}(3)$ & $120.8(3)$ & $\mathrm{C}(21)-\mathrm{C}(22)-\mathrm{N}(3)$ & $118.0(3)$ \\
$\mathrm{C}(22)-\mathrm{C}(23)-\mathrm{C}(24)$ & $117.2(4)$ & $\mathrm{N}(4)-\mathrm{C}(24)-\mathrm{C}(23)$ & $119.7(4)$ \\
$\mathrm{C}(15)-\mathrm{N}(1)-\mathrm{C}(19)$ & $123.0(2)$ & $\mathrm{C}(5)-\mathrm{N}(2)-\mathrm{C}(1)$ & $126.1(2)$ \\
$\mathrm{C}(5)-\mathrm{N}(2)-\mathrm{C}(17)$ & $116.8(2)$ & $\mathrm{C}(1)-\mathrm{N}(2)-\mathrm{C}(17)$ & $117.0(2)$ \\
$\mathrm{C}(10)-\mathrm{N}(3)-\mathrm{C}(14)$ & $125.8(2)$ & $\mathrm{C}(10)-\mathrm{N}(3)-\mathrm{C}(22)$ & $116.2(2)$ \\
$\mathrm{C}(14)-\mathrm{N}(3)-\mathrm{C}(22)$ & $117.8(2)$ & $\mathrm{C}(20)-\mathrm{N}(4)-\mathrm{C}(24)$ & $123.8(3)$ \\
\hline
\end{tabular}


Table S3. Hydrogen bonds of for compounds 1, 1P and $2\left(\AA\right.$ and $\left.{ }^{\circ}\right)$

\begin{tabular}{|c|c|c|c|c|}
\hline \multicolumn{5}{|c|}{ Compound 1} \\
\hline $\mathrm{D}-\mathrm{H} \cdots \mathrm{A}$ & $\mathrm{d}(\mathrm{D}-\mathrm{H})$ & $\mathrm{d}(\mathrm{H} \cdots \mathrm{A})$ & $d(D \cdots A)$ & $<(\mathrm{DHA})$ \\
\hline $\mathrm{C}(8)-\mathrm{H}(8) \ldots \mathrm{Cl}(4) \# 3$ & 0.93 & 2.80 & $3.561(4)$ & 139.7 \\
\hline $\mathrm{C}(11)-\mathrm{H}(11) \ldots \mathrm{O}(5)$ & 0.93 & 1.93 & $2.817(5)$ & 157.5 \\
\hline $\mathrm{C}(12)-\mathrm{H}(12) \ldots \mathrm{O}(2) \# 4$ & 0.93 & 2.29 & $3.004(4)$ & 133.7 \\
\hline $\mathrm{C}(22)-\mathrm{H}(22) \ldots \mathrm{Cl}(3) \# 5$ & 0.93 & 2.98 & $3.636(3)$ & 128.6 \\
\hline $\mathrm{C}(22)-\mathrm{H}(22) \ldots \mathrm{Cl}(4) \# 5$ & 0.93 & 2.91 & $3.598(3)$ & 132.0 \\
\hline $\mathrm{N}(2)-\mathrm{H}(2) \ldots \mathrm{Cl}(2) \# 6$ & 0.86 & 2.35 & $3.156(3)$ & 155.4 \\
\hline $\mathrm{N}(3)-\mathrm{H}(3) \ldots \mathrm{Cl}(1) \# 4$ & 0.86 & 2.71 & $3.310(4)$ & 127.8 \\
\hline \multicolumn{5}{|c|}{$\# 1-x+1,-y+1,-z ; \# 2-x,-y+2,-z+1 ; \# 3 x+1, y, z ; \# 4-x+1,-y+1,-z+1 ; \# 5 x, y+1, z ; \# 6$-x,-y+1,-z } \\
\hline \multicolumn{5}{|c|}{ Compound 1P } \\
\hline $\mathrm{D}-\mathrm{H} \cdots \mathrm{A}$ & $d(D-H)$ & $\mathrm{d}(\mathrm{H} \cdots \mathrm{A})$ & $d(D \cdots A)$ & $<(\mathrm{DHA})$ \\
\hline $\mathrm{C}(8)-\mathrm{H}(8) \ldots \mathrm{Cl}(4) \# 3$ & 0.93 & 2.79 & $3.557(3)$ & 140.1 \\
\hline $\mathrm{C}(11)-\mathrm{H}(11) \ldots \mathrm{O}(5)$ & 0.93 & 1.95 & $2.827(5)$ & 156.9 \\
\hline $\mathrm{C}(12)-\mathrm{H}(12) \ldots \mathrm{O}(2) \# 4$ & 0.93 & 2.29 & $3.013(3)$ & 134.2 \\
\hline $\mathrm{C}(22)-\mathrm{H}(22) \ldots \mathrm{Cl}(3) \# 5$ & 0.93 & 2.99 & $3.642(3)$ & 128.7 \\
\hline $\mathrm{C}(22)-\mathrm{H}(22) \ldots \mathrm{Cl}(4) \# 5$ & 0.93 & 2.90 & $3.591(3)$ & 132.1 \\
\hline $\mathrm{N}(2)-\mathrm{H}(2) \ldots \mathrm{Cl}(2) \# 6$ & 0.86 & 2.36 & $3.159(3)$ & 155.4 \\
\hline $\mathrm{N}(3)-\mathrm{H}(3) \ldots \mathrm{Cl}(1) \# 4$ & 0.86 & 2.70 & $3.306(3)$ & 128.4 \\
\hline \multicolumn{5}{|c|}{$\# 1-\mathrm{x}+1,-\mathrm{y}+1,-\mathrm{z} ; \# 2-\mathrm{x}+2,-\mathrm{y},-\mathrm{z}+1 ; \# 3 \mathrm{x}+1, \mathrm{y}, \mathrm{z} ; \# 4-\mathrm{x}+1,-\mathrm{y}+1,-\mathrm{z}+1 ; \# 5-\mathrm{x}+1,-\mathrm{y},-\mathrm{z}+1 ; \# 6 \mathrm{x}+1, \mathrm{y}, \mathrm{z}+1$} \\
\hline \multicolumn{5}{|c|}{ Compound 2} \\
\hline $\mathrm{D}-\mathrm{H} \cdots \mathrm{A}$ & $d(D-H)$ & $\mathrm{d}(\mathrm{H} \cdots \mathrm{A})$ & $d(D \cdots A)$ & $<(\mathrm{DHA})$ \\
\hline $\mathrm{O}(5)-\mathrm{H}(205) \ldots \mathrm{O}(4) \# 1$ & $0.798(10)$ & $2.37(3)$ & $3.013(4)$ & $138(4)$ \\
\hline $\mathrm{O}(5)-\mathrm{H}(205) \ldots \mathrm{Cl}(4)$ & $0.798(10)$ & $2.84(3)$ & $3.444(3)$ & $134(4)$ \\
\hline $\mathrm{O}(5)-\mathrm{H}(105) \ldots \mathrm{O}(1) \# 2$ & $0.807(10)$ & $2.20(3)$ & $2.904(4)$ & $146(5)$ \\
\hline $\mathrm{N}(4)-\mathrm{H}(4) \ldots \mathrm{Cl}(1) \# 3$ & 0.86 & 2.35 & $3.118(3)$ & 148.7 \\
\hline $\mathrm{N}(1)-\mathrm{H}(1) \ldots \mathrm{Cl}(2) \# 4$ & 0.86 & 2.56 & $3.300(3)$ & 144.2 \\
\hline $\mathrm{C}(24)-\mathrm{H}(24) \ldots \mathrm{Cl}(3) \# 5$ & 0.93 & 2.95 & $3.392(4)$ & 110.6 \\
\hline $\mathrm{C}(23)-\mathrm{H}(23) \ldots \mathrm{O}(5) \# 6$ & 0.93 & 2.37 & $3.215(5)$ & 150.1 \\
\hline $\mathrm{C}(21)-\mathrm{H}(21) \ldots \mathrm{O}(3) \# 2$ & 0.93 & 2.54 & $3.206(4)$ & 129.2 \\
\hline $\mathrm{C}(20)-\mathrm{H}(20) \ldots \mathrm{Cl}(3) \# 3$ & 0.93 & 2.83 & $3.510(3)$ & 131.1 \\
\hline $\mathrm{C}(18)-\mathrm{H}(18) \ldots \mathrm{O}(2) \# 4$ & 0.93 & 2.54 & $3.231(3)$ & 131.1 \\
\hline $\mathrm{C}(16)-\mathrm{H}(16) \ldots \mathrm{O}(5)$ & 0.93 & 2.44 & $3.348(4)$ & 167.2 \\
\hline $\mathrm{C}(15)-\mathrm{H}(15) \ldots \mathrm{Cl}(4)$ & 0.93 & 2.71 & $3.462(4)$ & 138.3 \\
\hline $\mathrm{C}(15)-\mathrm{H}(15) \ldots \mathrm{Cl}(1)$ & 0.93 & 2.69 & $3.369(3)$ & 130.9 \\
\hline$\# 1 \mathrm{x},-\mathrm{y}, \mathrm{z}-1 / 2 ; \# 2 \mathrm{x}, \mathrm{y}-1, \mathrm{z}$ & $2, y-1 / 2, z+1$ & $1, z ; \# 5 \mathrm{x}+1 /$ & $+1 ; \# 6 \mathrm{x},-\mathrm{y}$ & \\
\hline
\end{tabular}

